\title{
Model Predictive Control for Field Excitation of Synchronous Generators
}

\author{
Thomas Øyvang ${ }^{1}$ Bernt Lie $^{1} \quad$ Gunne John Hegglid ${ }^{2}$ \\ ${ }^{1}$ Faculty of Technology, University College of Southeast, Norway, \{Thomas. Oyvang, Bernt.Lie\}@usn.no \\ ${ }^{2}$ Skagerak Energi AS, Norway, GunneJohn. Hegglideskagerakenergi.no
}

\begin{abstract}
This paper describes a Model Predictive Control (MPC) system for voltage control through field excitation of hydroelectric generating units. An attractive feature of MPC is its capability to handle Multiple Input, Multiple Output (MIMO) systems and nonlinear systems taking constraints into account. The system under study is a power system based on detailed models from Matlab's SimPowerSystems ${ }^{\mathrm{TM}}$ and parametrized according to the Nordic model from the Norwegian Transmission System Operator (TSO), Statnett. The primary role of the field excitation control system is to quickly respond to voltage disturbances occurring in the power system. The control system is tested for both first-swing transient stability and long term voltage stability.
\end{abstract}

Power system modeling and control, model predictive control, SimPowerSystems, first-swing rotor enhancement, long term voltage stability, fmincon

\section{Introduction}

In this paper a Model Predictive Controller (MPC) for field excitation of synchronous generators is tested, and compared to a classical controller typically used for this purpose. MPC is an advanced control methodology that has proved to be successful in real-life applications. An attractive feature of MPC is its capability to handle Multiple Input, Multiple Output (MIMO) systems and nonlinear systems taking constraints into account (Maciejowski, 2002).

There are mainly two requirements for successful operation of a power system (Hegglid, 1983). The first requirement of reliable service is to keep the generators running in parallel (synchronous) and with necessary capacity to meet load demand. If at any time the generator loses synchronism with the rest of the system, significant voltage and current fluctuations may occur and transmission lines may be automatically tripped by their relays at undesired locations (Kundur, 1994).

A second requirement of reliable service is to maintain the integrity of the power network. Interruptions in this network may hinder flow of power to the load, leading to severe blackouts of the power system. This usually requires a study of large geographical areas since almost all power stations and load centres are connected in one system.

A controller should maintain both of these requirements: keeping the generators running in parallel and to maintain the integrity of the power system. This is achieved mainly by reducing the first swing of the rotors of the synchronous generators after large disturbances, and the damping of power oscillations (also small disturbances). Another important requirement for a controller is to provide necessary reactive power supply $Q$ for enhancement of voltage stability. Reactive power can be used to compensate for voltage drops, but must be provided closer to the demands than active power $P$ needs due to transportation limitations of reactive power through the grid.

A flexible power factor control on large synchronous generators located close to points of high demand could enhance the voltage stability of a power system. The Norwegian Network Code FIKS ${ }^{1}$ states that synchronous generators $\geq 1$ MVA must connect to the grid with a $\cos \phi$ $\geq 0.86$ overexcited and $\leq 0.95$ underexcited at maximum load (independent of the location from the point of demand). However, necessary enhancement of voltage stability could be secured through use of more advanced control where more reactive power is temporary available from large generators generated locally.

The paper is organized as follows. Section 2 provides a brief overview of field excitation control of synchronous generators. Section 3 describes MPC for the excitation system and the modeling workflow used in this paper. Section 4 describes MPC tuning. Section 5 introduces, describes and discuss the different tests and results from simulations. In Section 6, conclusions and future perspectives are presented.

\section{Field Excitation Control}

\subsection{Capability Curve}

Synchronous machines are capable of producing and consuming reactive power. When the machine is overexcited, it generates $Q$ and deliver it to the power system. Negative $Q$ then, flows from the system into the machine to maintain its magnetization when its own field is underexcited. Generally, reactive power support is divided into

\footnotetext{
${ }^{1}$ Funksjonskrav I Kraft-Systemet 2012 from the Norwegian Transmission System Operator (TSO) (Statnett, 2012)
} 
two categories: static and dynamic (Kundur, 1994). Dynamic reactive power is produced from equipment that can quickly change the $Q$ independent of the voltage level such as a synchronous generator or condenser (generator without active power exchange $P$ with the grid). Thus, the equipment can increase its reactive power production level when the voltage drops, and prevent a voltage collapse.

A generators operating constraints can be visualized through a capability diagram (Farnham and Swarthout, 1953). In Figure 1, the capability curve is shown for the synchronous generator used for simulations in this paper.

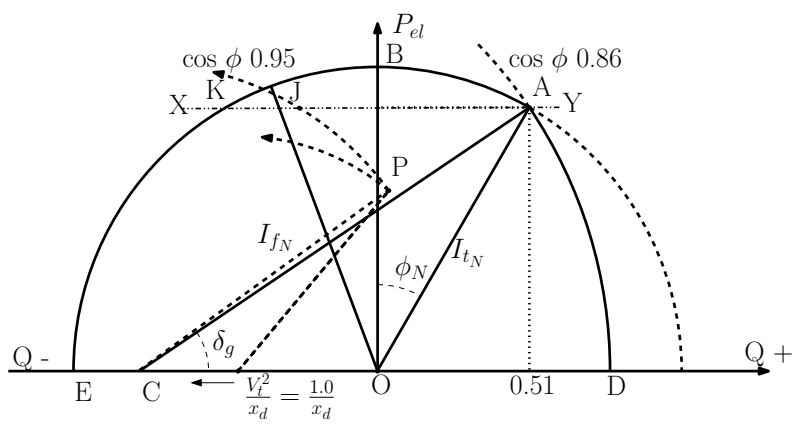

Figure 1. Capability curve for synchronous generator used in simulations. Point $A$ represents the name-plate rated conditions with power factor $\cos \phi=0.86$ for the generator. Rated (nominal) MVA of the machine $S_{N}=\sqrt{P_{N}^{2}+Q_{N}^{2}}$ is taken as 1.0 per unit on its own rated MVA base. Hence, the rated conditions for machine operation are 0.86 per unit MW and 0.51 per unit $\operatorname{MVAr}(\cos \phi=0.86, \sin \phi=0.51)$.

Point A is just one point in a rather extensive area of Figure 1. Few machines are operated at any length of time exactly in the condition of A. The operating conditions in overexcited mode is bounded by the armature current limit $\mathrm{AB}$ (circle having its centre at $\mathrm{O}$, and radius equal to rated armature current, $I_{t_{N}}$ or OA ) and the field current limit $\mathrm{AD}$ (circle having its centre at $\mathrm{C}$, and radius equal to rated full load field current, $I_{f_{N}}$ or CA). The controller can control the field current represented by $\mathrm{CP}$ in Figure 1. $\mathrm{CP} / \mathrm{CA}$ is simply the proportion of rated field current, and this is just the amount necessary to permit the machine to handle the $P$ and $Q$ represented by point P. If the load increases without any change in the field current, this causes a movement of operating point $\mathrm{P}$ along the arc PK. This path runs almost directly into the instability region (Farnham and Swarthout, 1953) of the generator, e.g minimum field current for stable operation. The final operating point would lie on the XY line, representing limits set by the turbine power. The figure also shows the effect of increased synchronous reactance $x_{d}$ of the machine. This increase in $x_{d}$ makes the machine reach the stability limits even faster. The maximum reactive power that can be delivered from this generator is defined by $\mathrm{OD}=\mathrm{CA}-\mathrm{CO}$ (e.g. as an synchronous condenser). With a synchronous reactanse $x_{d}$ of 1.24 in per-unit, terminal voltage $V_{t}=1.0$ in per-unit, and the given operating conditions in Figure 1, the rated field current ${ }^{2}$ can be calculated to be 1.6 from

$$
I_{f_{N}}=\sqrt{\left(\frac{V_{t}^{2}}{x_{d}}+\frac{Q_{t}}{S_{N}}\right)^{2}+\cos \phi_{N}^{2}} .
$$

\subsection{Classical Control}

The excitation (or field) current required to produce the magnetic field inside the generator, is provided by the exciter and is controlled by an Automatic Voltage Regulator (AVR) (Kundur, 1994). The AVR regulates the generator terminal voltage by controlling the amount of current supplied to the generator field winding by the exciter. Power System Stabilizers (PSS) are feed-forward supplementary control devices which are installed in generator excitation systems to increase damping of (power) oscillations. The specification of excitation systems is guided by IEEE standards 421 (IEEE, 2007). In this paper, a synchronous machine voltage regulator and exciter based on the IEEE type ST1A excitation and Kundur's (Kundur, 1994) generic PSS is used. This type represents a classical exciter model of static potential-source controlled-rectifier systems. This classical control structure is shown in Figure 2

There are two key factors that define an excitation system: the transient gain and the ceiling force ratio (IEEE, 2007). Because of a very high field forcing capability of the system, a field current limiter is employed to protect the generator rotor and exciter. The transient gain has a direct impact on small signal and dynamic stability. Too small a value may fail to give the desired performance, while too high a value (faster response) may result in instability during faults.

\section{Concept and Formulation of MPC}

MPC is an algorithm where an optimal control problem is solved at the current time, then a receding/sliding horizon technique is applied as time progresses. The predictive controller considers both past situations (given by state) and the changing of the system in a finite future time horizon. To solve the optimal control problem, an optimization routine is needed. In the optimization problem, an objective/criterion to be maximized/minimized is formulated together with constraints (Maciejowski, 2002).

Optimal control is an open loop optimization problem. If input disturbances and references change in the future, the controller has no knowledge about this change since no feedback is presented in the solution. Under such conditions optimal control problems may give good performance. To come around this challenge, feedback is introduced to the optimal controller. A way to do this is called Model Predictive Control (MPC) (Sharma, 2014).

\footnotetext{
${ }^{2}$ The rated field current is the direct current in the field winding of a machine, when operating at rated voltage, current and speed and at rated power factor for synchronous machines (Farnham and Swarthout, 1953).
} 


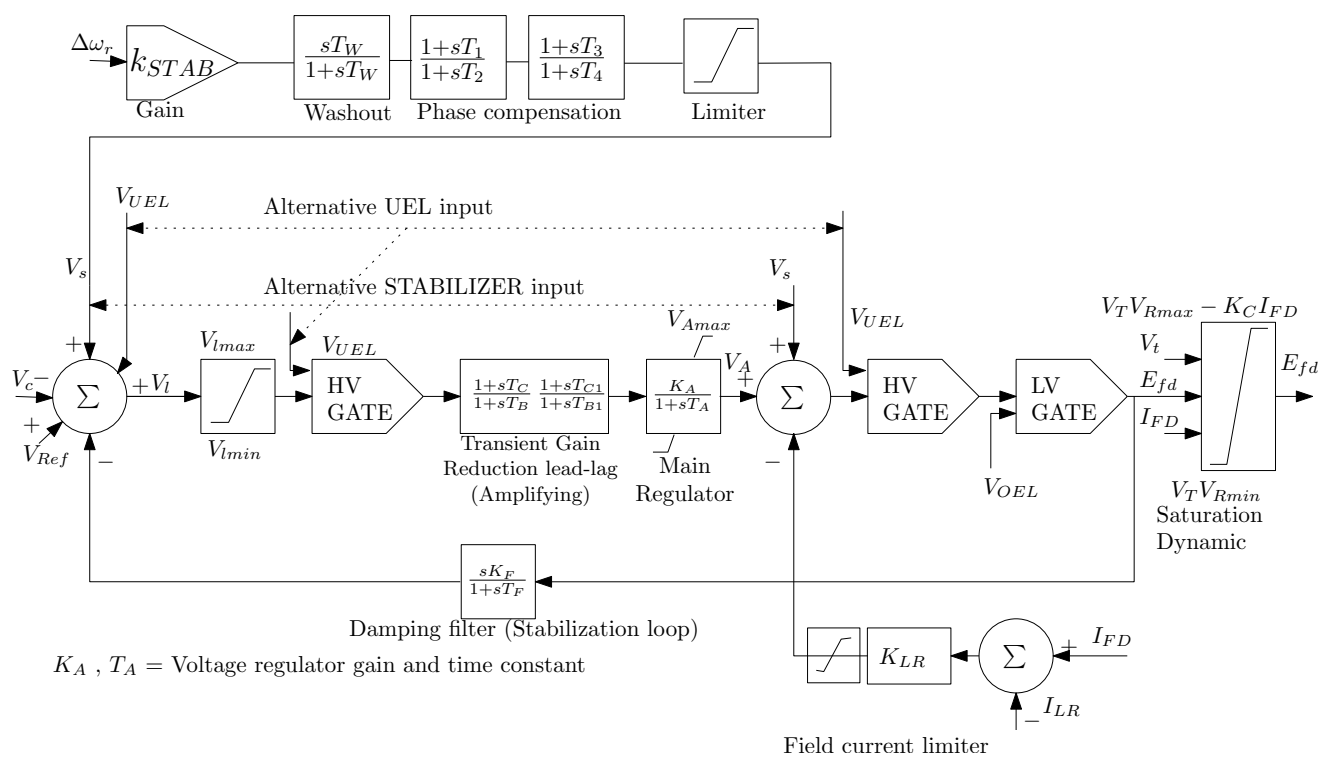

Figure 2. The classical static excitation control ST1A with PSS used in simulations. The PSS representation consist of a phase compensation block, a signal washout block and a gain block. The models are tuned according to Kundur (Kundur, 1994).

As mentioned above, MPC is optimal control with a sliding horizon strategy, e.g. a new optimal control problem is solved at every time step $\Delta t$.

Assume the initial state of the plant $x_{j}$ is known, together with current/future references $r_{j+1}, r_{j+2}, . ., r_{j+N}$ and disturbances $w_{j}, w_{j+1}, . ., w_{j+N-1}$. By solving the optimal control problem, this leads to an optimal open loop control input sequence $u_{j \mid j}^{*}, u_{j+1 \mid j}^{*}, . ., u_{j+N-1 \mid j}^{*}$. Subscript $k \mid j$ implies the control input at time $k$, when current state $x_{j}$ is known. It is clear that $u_{k \mid j}^{*}=$ $u_{k}^{*}\left(x_{j}, r_{j+1}, . ., r_{j+N}, w_{j}, . ., w_{j+N-1}\right)$. The dependence of $u_{k \mid j}^{*}$ on $r_{j+1, . .}, r_{j+N}, w_{j}, . ., w_{j+N-1}$ gives feed forward from $r_{j+1}, . ., r_{j+N}, w_{j}, . ., w_{j+N-1}$. However, optimal control where $u_{k}=u_{k \mid j}^{*}$ does not give feedback since $u_{k}$ only depends on $x_{j}$. To have feedback, we require that $u_{k}$ depends on $x_{k}$. To achieve feedback, introduce receding horizon: set $u_{j}=u_{j \mid j}^{*}$. Then find or estimate $x_{j+1}$ after $u_{j}$ has been injected, shift/recede the optimal control horizon one step. The process is then repeated to compute $u_{j+1 \mid j+1}^{*}, u_{j+2 \mid j+1}^{*}, . ., u_{j+N+1 \mid j+1}^{*}$ by setting $u_{j+1}=$ $u_{j+1 \mid j+1}^{*}$ and we introduce feedback.

Because of feedforward the controller can react before known disturbances (or set point changes) affect the process. A model of the disturbance should then be included along with the model of the process while solving the optimal control problems at each time step.

\subsection{MPC as Excitation Control}

For the power system generator excitation control, the selected system outputs are the generator voltage and angular velocity. The manipulating input, $u$, is the generator field excitation voltage $E_{f d}$ when it is used as a primary controller. Alternatively MPC is also used as a secondary controller to change set-point of the classical (primary) controller $u=V_{\text {ref }}$. In Figure 3, the control structure used in the simulations is shown. For transient stability, MPC works as primary control while during long-term stability analysis MPC was tested both for primary and secondary control. The control system can also change between classical and MPC control during simulation.

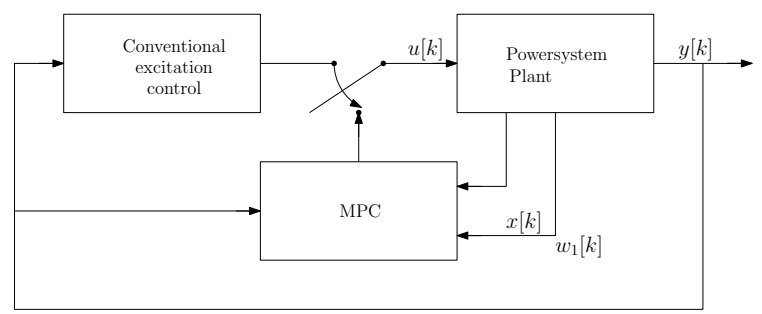

Figure 3. Structure of plant and controller. Here, MPC is a Multiple Input, Single Output (MISO) controller.

The performance index $J$ is scalar and is computed as a summation of the square of the deviations between outputs and references, $\Delta e$, and the variations of the controlling inputs to the controlled system, $\Delta u_{c}$. Weighting factors $\mathbf{Q}, \mathbf{P}$ and $\mathbf{R}$ are included in $J$ as tuning parameters.

$$
J=\sum_{j=1}^{N_{p}} \Delta e_{V, j}^{T} \mathbf{Q} \Delta e_{V, j}+\Delta e_{\omega_{, j}}{ }^{T} \mathbf{P} \Delta e_{\omega_{, j}}+\sum_{j=1}^{N_{c}} \Delta u_{c, j-1}{ }^{T} \mathbf{R} \Delta u_{c, j-1}
$$

where

$$
\begin{aligned}
\Delta u_{c, j} & =u_{c, j}-u_{c, j-1} \\
\Delta e_{\omega_{, j}} & =\omega_{r e f}-\omega_{, j} \\
\Delta e_{V, j} & =V_{r e f}-V_{t, j}
\end{aligned}
$$

The control input is the field excitation voltage

$$
u_{c_{j}}=\left[E_{f d_{c_{j}}}\right]
$$


$N_{p}$ and $N_{c}$ are prediction and control horizon lengths. The inequality constraint on the controlled variable are $E_{f d_{c}} \in\left[E_{f d_{\min }}, E_{f d_{\max }}\right]$ where $E_{f d_{\text {min }}}=-3.2$ per-unit and $E_{f d_{\text {max }}}=+3.2$ per-unit (Two times the rated field current according to FIKS (Statnett, 2012)).

The control output

$$
y_{j}=\left[V_{t, j}, \omega_{g, j}\right]^{T}
$$

\subsection{Modeling and Control Workflow}

The modeling part in this paper is done with the powerlib library of SimPowerSystems ${ }^{\mathrm{TM}}$ built on the Simscape $^{\mathrm{TM}}$ language, running within the Simulink $\mathrm{R}$ environment. SimPowerSystems provides component libraries and analysis tools for modeling and simulating electrical power systems. Since Simulink uses MATLAB as its computational engine, MATLAB toolboxes are available. In this paper, MPC is implemented using the MATLAB Optimization Toolbox and the fmincon solver with the Active-Set method.

For simulations, the phasor domain solution method with Simulink@variable-step solvers (ode23t and ode23tb) are used. The phasor solution method is mainly used to study electromechanical oscillations of power systems, which is the case study in this paper.

To further increase the speed of optimization, all simulations were done in Accelerator mode and with the Fast Restart command. The Accelerator mode generates and links code into a C-MEX S-function. The idea of Fast Restart is to perform the model compilation once and reuse the compiled information for subsequent simulations. Also the SimState function was used for easily integration within the optimization algorithm. A SimState is the snapshot of the state of a model at a specific time during simulation.

\section{Tuning the MPC Controller}

\subsection{Time Response}

The voltage controller needs to operate freely and without unnecessary restriction within performance limits of the generator and excitation system. A standard procedure for evaluating the response of the closed-loop excitation control system is to document its dynamic characteristics. A small-signal performance measure is expressed in terms of indices associated with time response. A stepresponse test is done on the regulator in open circuit conditions according to TSO. The mathematical model design to be used for the MPC tuning and also first-swing tests is a classical SMIB (Singel Machine Infinite Bus). This model consist of a three-phase salient-pole synchronous machine modelled in the dq rotor reference frame, threephase transformer, transmission line and a voltage source as an inifinite bus as shown in Figure 4.

The model is a power plant in Norway: the 175 MVA hydro power plant Kobbelv aggregate 2 owned by Statkraft AS (G2 in Area 2 in Figure 11). The main data for this generator is given in Table 1

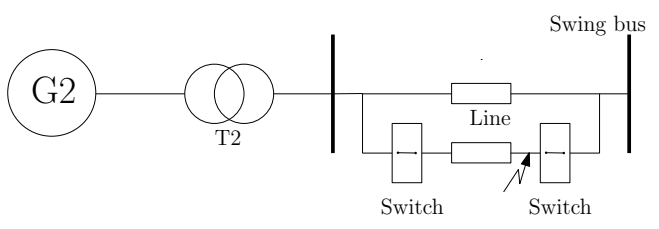

Figure 4. Singel Machine Infinite Bus (SMIB) for transient stability simulations.

Table 1. Main data for $G 2$ in area 2.

\begin{tabular}{llll}
\hline \hline Description & Parameter & Value & Unit \\
\hline \hline Rated power & $S_{N}$ & 175 & MVA \\
Rated Power factor & $\cos \phi_{N}$ & 0.86 & \\
Rated voltage & $V t_{N}$ & 16.5 & $\mathrm{kV}$ \\
Frequency & $f$ & 50 & $\mathrm{~Hz}$ \\
Number of polepairs & $p$ & 8 & \\
Inertia constant & $H$ & 2.9 & $\mathrm{~s}$ \\
\hline \hline
\end{tabular}

\subsection{Open Circuit Conditions}

The voltage regulator should be verified by the impulse response in open circuit conditions. For the voltage regulator, a 5\% up and down step-response test on the voltage regulator should be carried out. The voltage should be measured over the generator terminals and the response should be non-oscillatory with a overshoot less than $15 \%$ of the impulse response itself. The results are shown in Figure 5.

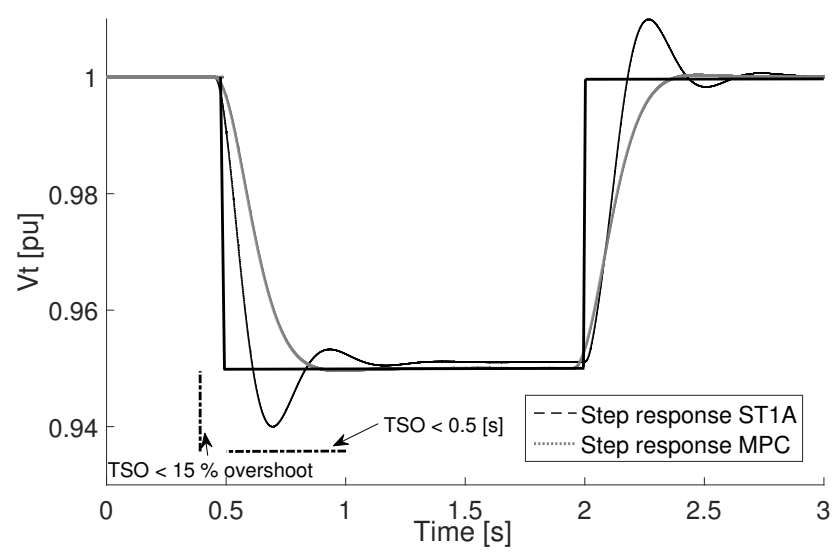

Figure 5. The figure is showing a open circuit small-signal time response test with respect to TSO requirement for both MPC and classical control ST1A. The settling time must be $<0.5$ seconds for a 0.95 to 1 step-up and a 1 to 0.95 step-down from steady state value according to FIKS (Statnett, 2012). The overshoot shall be less than $15 \%$ of the change. The MPC tuning parameters were $N_{p}=5, N_{c}=1, \mathbf{Q}=300, \mathbf{P}=5, \mathbf{R}=0.01$ and $\Delta t=0.05$. 


\section{First-swing Angle Stability En- hancement}

First-swing angle stability (or transient stability) enhancement is necessary to avoid loss of synchronism. Two factors which indicate the stability are the angular swing (during and after a fault) and the critical clearing time $t_{c r i t}{ }^{3}$ (or clearing angle $\delta_{\text {crit }}$ ) of a fault (Grainger and Stevenson, 1994). The generator rotor angle swing normally peaks between 0.4 and 0.75 seconds. This short time demands a fast acting voltage regulator to boost the internal voltage through the field excitation. The steady-state power-angle characteristic presented in Figure 6 shows the highly non-linear relationship between interchange electrical power $P_{e}$ and angular position $\delta$ of the rotors of the synchronous generators (The effect of AVR and damping windings (Kundur, 1994) are neglected).

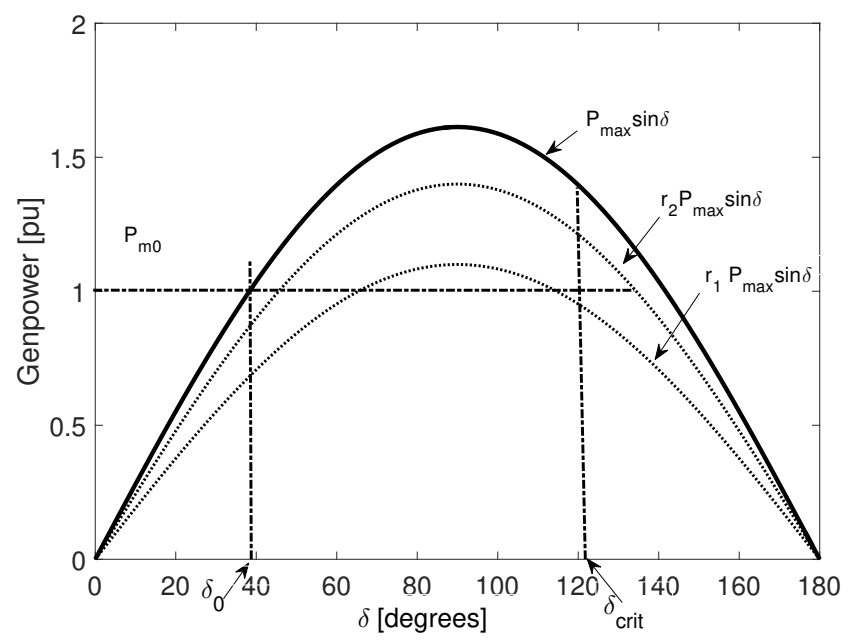

Figure 6. Plot of power-angle curves (Pre-fault $P_{\max }$, during fault $r_{1} P_{\max }$ and post-fault $r_{2} P_{\max }$ ) for synchronous generator G2 Area 2 showing, initial mechanical power and electrical power $P_{m 0}=P_{e}=1 p u$, inital angular position of the rotor $\delta_{0}=39 \mathrm{de}-$ grees and the critical clearing angle calculated to $\delta_{\text {crit }}=122 \mathrm{de}-$ grees and corresponding critical clearing time $t_{c r i t}=0.28 \mathrm{sec}-$ onds.

Position $\delta_{0}<90$ in Figure 6 is the initial operating point of a stable operation. The swing equation for the machine with constant flux linkage may be written in acceleration form as

$$
\begin{gathered}
\frac{H}{180 f} \frac{d^{2} \delta}{d t^{2}}=P_{m}-P_{e}=1.0-P_{\max } \sin \delta \\
P_{\max }=\frac{E_{g} \cdot V_{\text {bus }}}{x_{s}}
\end{gathered}
$$

$E_{g}$ is the synchronous machine transient voltage, $x_{s}$ is the series transfer reactance between $E_{g}$ and swing bus $V_{b u s}$, and $f$ is the electric frequency. In all the simulations,

\footnotetext{
${ }^{3}$ The critical clearing time is the maximum elapsed time from the initiation of the fault until its isolation such that the power system is stable.
}

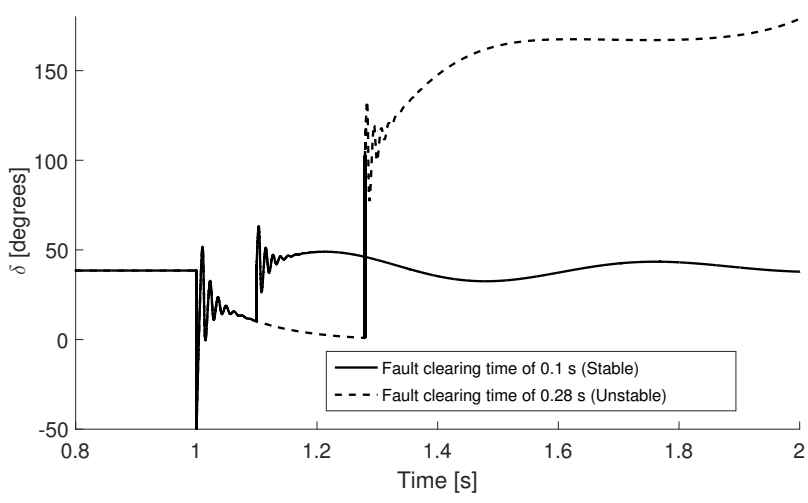

Figure 7. Plot of rotor angle $\delta$ affected by a three-phase fault under constant field voltage control for synchronous generator G2 in Area 2. The plot shows two different fault clearing times. The fault cleared after 0.28 seconds lead to loss of synchronism as calculated from $t_{\text {crit }}$.

$P_{m}$ is kept constant. When $P_{m}$ equals $P_{e}$, the machine operate at steady state synchronous speed. Both the inertia constant $H$ and transient reactance of the machine $x_{d}^{\prime}$ has a direct impact on the first swing (transient) studies. A smaller $H$ gives a larger angular swing. $P_{\max }$ decreases as the transient reactance increases since it forms a part of the overall series reactance. A decreased $P_{\max }$ constrains the machine to swing through a smaller angle from its original position before it reaches the critical clearing angle.

The MPC controller was tested and compared to classical control as shown in Figure 8 and Figure 9.

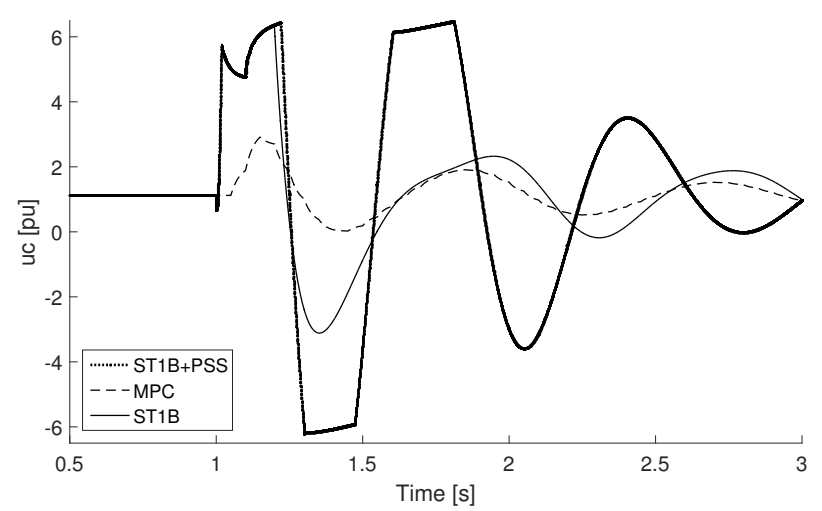

Figure 8. Change in field voltage $E_{f d}$ as a function of time under disturbance of a three-phase arc fault for three different types of control actions. Clearing time was 0.1 second. Future disturbance is not known for the controller.

\section{Long-term Voltage Stability En- hancement}

\subsection{Steady-state Voltage Stability}

The steady-state voltage-power characteristics (also called onion surface) shown in Figure 10 for the SMIB, gives insight into the voltage stability problem (Larsson, 2000). 


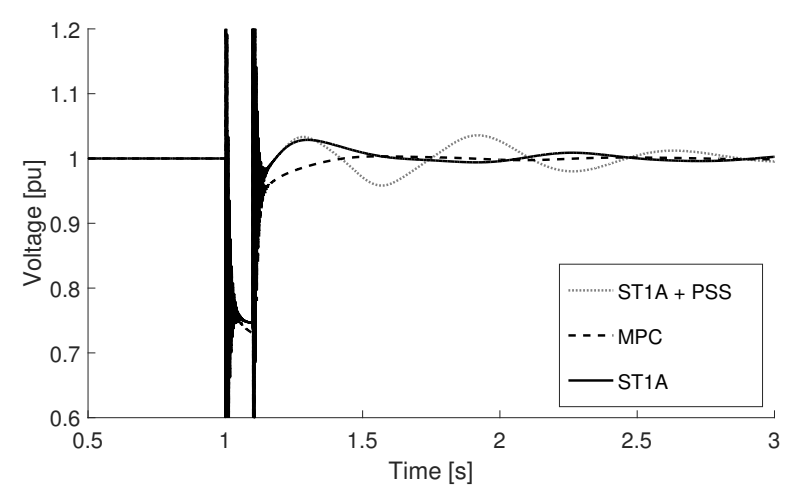

Figure 9. Change in terminal voltage $V_{t}$ as a function of time under disturbance of a three-phase arc fault for three different types of control actions. Clearing time was 0.1 second. Future disturbance is not known to the controller.

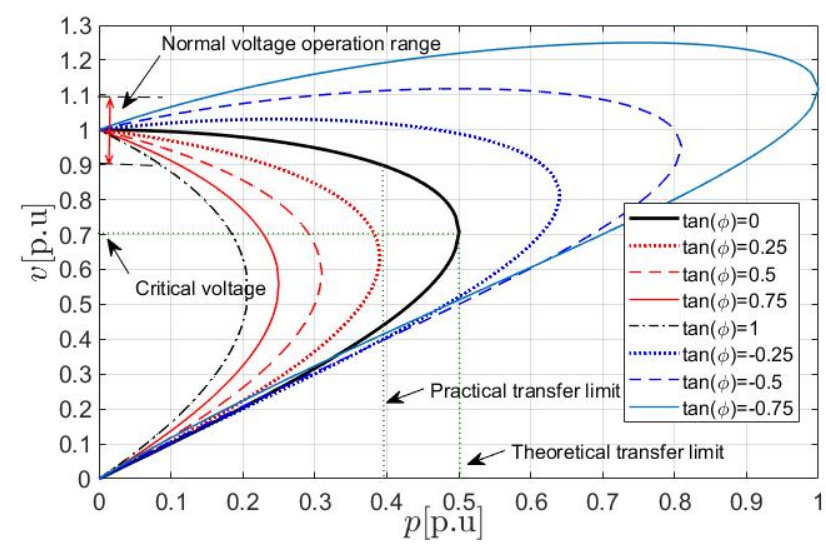

Figure 10. Normalized power $(p)$-voltage $(v)$ curves (onion surface) for steady-state voltage stability analysis. The practical and theoretical transfer limits and the critical voltage is given for $\tan (\phi)=0$.

The critical voltage $V_{c}$ (voltage collapse) points out the theoretically stability limit of the SMIB. The most important factor to provoke a voltage collapse (blackout) is the load model as presented in (yvang et al., 2014) . A widely used dynamic load characteristic is an exponential load model with fractional load exponents combined with time constants for both active and reactive power (Cutsem and Vournas, 1998). If the voltage is lower than a specified value, the load impedance is kept constant. An impedance load model adapts to the voltage. However, a dynamic load that recovers over time combined with automatic tap-changers would stress the power system. When load recovers in a highly loaded system, the need for reactive power increases with $I$. This will automatically bring the system to the edge. In addition one needs to make sure that generator exciters are limited (Kundur, 1994).

\subsection{Power System Simulator}

Large-disturbance (e.g. loss of load or loss of generation) long term voltage stability simulations requires the examination of the dynamic performance of the system over a period of time sufficient to capture the interactions of such as tap-changers and field current limiters (Kundur, 1994). This means that it is not only sufficient to simulate a three phase fault with clearing of fault after some milliseconds as done with first swing simulations. After an arc fault the system will either be transiently unstable, partly or as a whole (collapse), or it will return to a stable point. Thus, additionally outage of a line or any reactive power source is of interest (Cutsem and Vournas, 1998). For Long Term Voltage Stability (LTVS) simulations, a more complex simulator was needed to test the MPC controller in contrast to the SMIB simulator in transient studies. A Dynamic Study Model has been developed based on the Nordic Model 2010 High Load Case (Norgesmodellen) from Statnett. The geographical area is in the North region of Norway containing four synchronous generators and one synchronous compensator. It also contains an tap-changer connected to a dynamic load for stressing the system. The model was tuned to operate near its capacity limit and could exhibit cascading failures which could lead to blackouts. The power system model is shown in Figure 11.

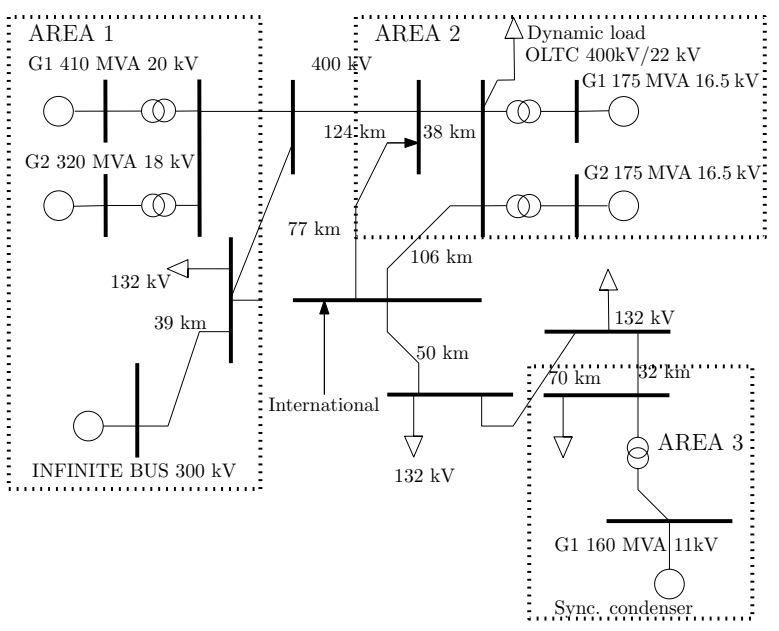

Figure 11. Power system simulator for long term voltage stability simulations based on the Nordic Model from Statnett. The system consists of three different Areas including four synchronous generators and one synchronous condenser. Power is also fed through the international transmission link.

\subsection{LTVS Simulations}

For LTVS simulations in this paper the international transmission link is disconnected demanding necessary reactive power to be delivered locally. The tap-changer is then trying to restore the voltage at load forcing the system to collapse. As expected from the steady state calculations, the power system have a blackout when part of the system is reaching the critical voltage limit around 0.7 per-unit as seen in Figure 12.

Results from simulations with MPC as primary control are shown in Figure 13 for different tuning parameters. Optimization is done at every $\Delta t=1 \mathrm{~s}$. A smaller $\Delta t$ and a longer prediction horizon $N_{p}$ gives better control but has a 


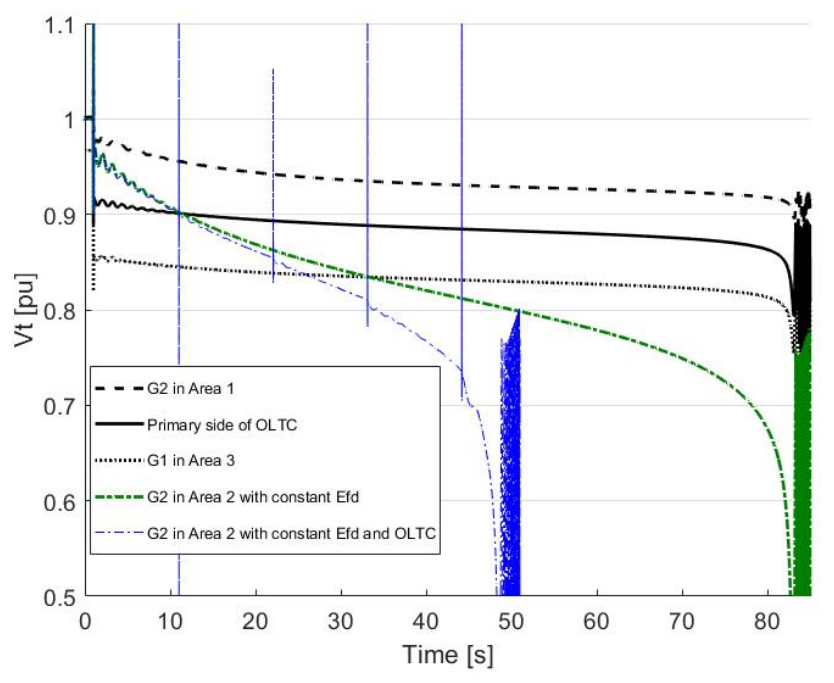

Figure 12. Voltage as a function of time for some selected buses in the power system with and without OLTC control on dynamic load. Area 1 Generator 1 and Generator 2 has constant field voltage. Area 2 Generator 1 and Area 3 Generator 1 has implemented classical control.

higher computational cost. The weighting factors are the same as earlier.

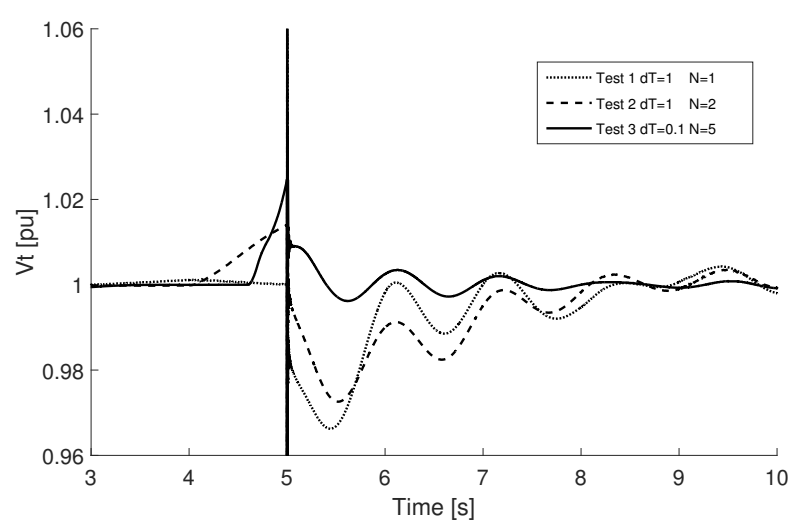

Figure 13. Voltage as a function of time where MPC control action are implemented at Area 2 G2 as primary control.

According to the TSO, the set-point voltage of the controller has to be between 0.9 and 1.05 per-unit value and can be set both locally and from a control center. For simulating such a scenario, MPC is used to change the set-point value $u=V_{r e f}$. In this case, the set-point was changed for both generators in Area 2 for bringing the voltage at the On-Load-Tap Controller (OLTC) bus closer to 1 pu after the disturbance. In this simulation, saturation is also included in the dynamic model. Future disturbance is also known. Results are shown in Figure 14 and Figure 15 with different $\Delta t$, horizon $N_{p}$ and tuning parameters.

\section{Discussion}

This investigation on transient and long term voltage stability considers the ideal (though unrealistic) situation where the internal MPC model exactly matches the real system (perfect MPC model). It is unrealistic to expect that the MPC controller could maintain a complete, accurate system representation. The degree to which MPC can tolerate model inaccuracy is core to practical power system implementation (Gong, 2008). A more realistic implementation could be an SMIB model representing the whole grid. Thus, state estimation should be done on the synchronous generator. In addition, the transfer reactance $x_{s}$ in (9) needs to be estimated and the infinite bus voltage $V_{\text {bus }}$ in (9) needs to adapt the actual voltage level in the system for a good predictive control action. The size of the inductor could be identified from the electromechanical first swing oscillations after a fault.

In these simulations, the full nonlinear model was used with MPC e.g. NMPC. NMPC requires extensive computing power to solve nonlinear constrained optimization problems in real time. In the case of transient stability, NMPC is too slow to avoid loss of synchronism. One other problem that occurs in simulating electrical circuits is that their equations often exhibit stiffness. In SimPowerSystems snubber resistors and capacitors are used across the switches to improve numerical stability. Ode 23tb was the fastest integrator in these simulations. However, with long term voltage stability when the MPC is acting only as set-point changing controller with a sampling time e.g every 5-10 second, NMPC could be feasible. In that case, a linear prediction model combined with C-code implementation should be used.

The MPC should also be tested for low frequency electromechanical oscillations in large interconnected power systems (e.g. both inter-area and local oscillations) (Kundur, 1994). This would determine more precise MPC tuning parameters applicable for both small- and large-signal stability.

The Simscape language is an object-oriented language based on MATLAB and is very attractive to use for power system modeling. One alternative is to integrate the open source object oriented Modelica modeling language with MATLAB through the FMI toolbox from Modelon. In that case the MATLAB optimization toolbox can be used to run MPC on a Modelica model through C code generation.

\section{Conclusion}

This paper investigates the use of Model Predictive Controll (MPC) for voltage (excitation) control of synchronous generators to enhance the stability of the power system. Simulation results show that a well tuned predictive controller combined with an internal model that exactly matches the real system, gives improved control action in all the simulations compared to classical control. Due to computational limitations, real time simulation for transient stability would not be possible with the strategy 


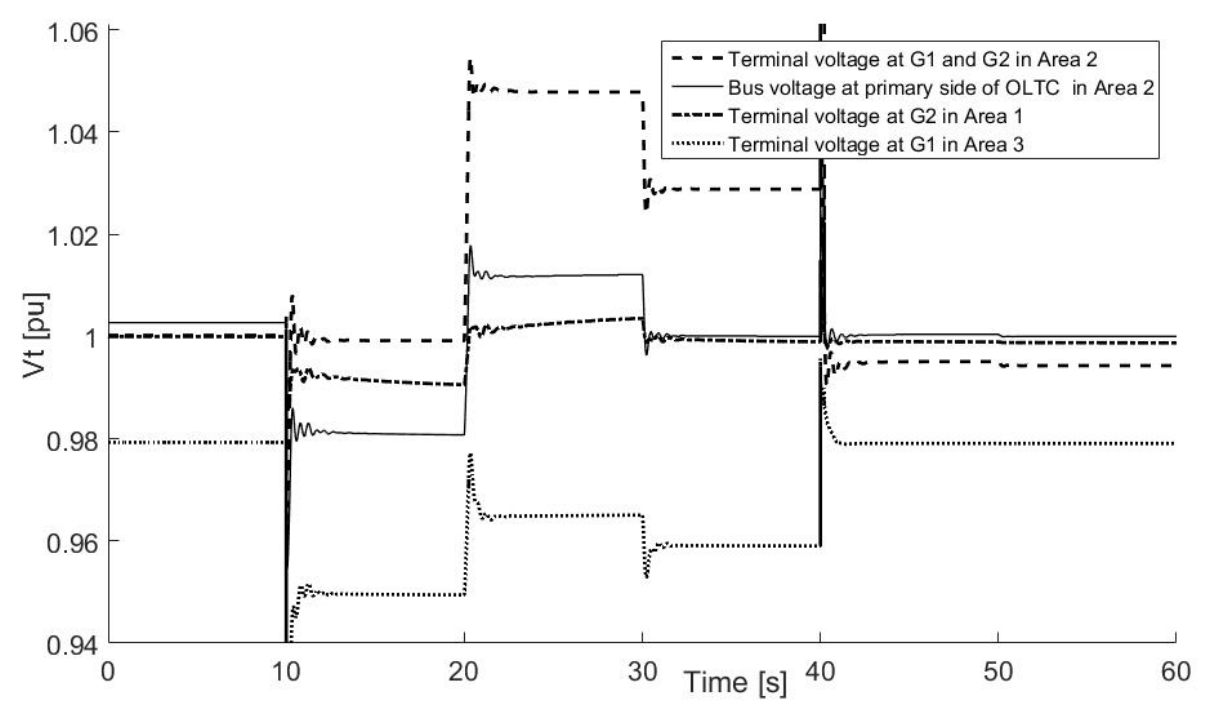

Figure 14. Voltage as a function of time where MPC is changing the set-point on classical control at G1 and G2 in Area 2 to bring voltage at primary side of OLTC bus closer to $1 \mathrm{pu}$. Optimization is done at every $\Delta t=10 \mathrm{~s}$ and the horizon is $N_{p}=1$. The international transmission line in Figure 11 are disconnected at 10 seconds and reconnected at 40 seconds in this simulations. The MPC tuning parameters were $\mathbf{Q}=300, \mathbf{P}=5, \mathbf{R}=0.01$.

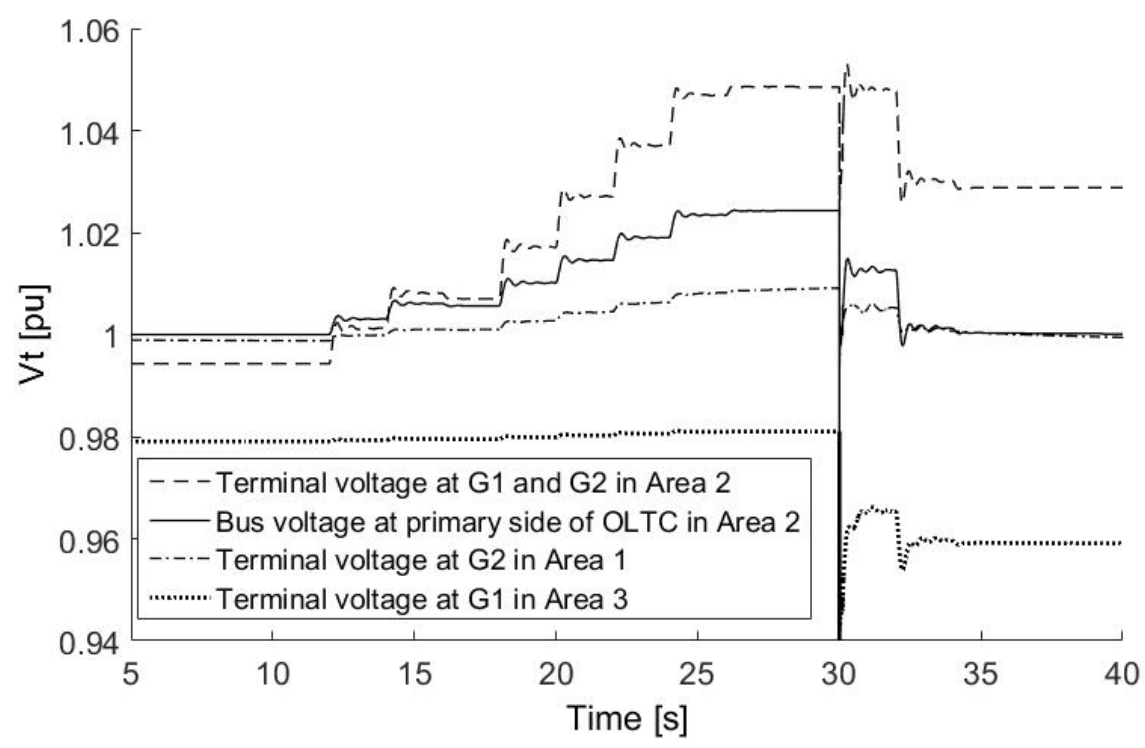

Figure 15. Voltage as a function of time where MPC is changing the set-point on classical control at G1 and G2 in Area2 to bring voltage at primary side of OLTC bus closer to $1 \mathrm{pu}$. Optimization is done at every $\Delta t=2 s$ and the horizon is $N_{p}=10$. The international transmission line in Figure 11 are disconnected at 30 seconds in this simulations. The MPC tuning parameters were $\mathbf{Q}=30, \mathbf{P}=1, \mathbf{R}=1$. 
presented in this paper. However, a reduced power system model combined with $\mathrm{C}$-code generation could be a feasible solution for faster NMPC action.

\section{Acknowledgment}

The financial support from Statkraft ASA of the PhD study of the first author is greatly acknowledged. The practical support from Jan Petter Haugli, Statkraft ASA is likewise acknowledged.

\section{References}

T. V. Cutsem and C. Vournas. Voltage Stability of Electric Power Systems, volume 441. Springer US, 1998.

S. B. Farnham and R. W. Swarthout. Field excitation in relation to machine and system operation [includes discussion]. Transactions of the American Institute of Electrical Engineers. Part III: Power Apparatus and Systems, 72(2), Jan 1953. ISSN 0097-2460. doi:10.1109/AIEEPAS.1953.4498759.

B. Gong. Voltage stability enhancement via model predictive control. PhD thesis, The University of Wisconsin, Madison, 2008. ProQuest Dissertations and Theses, 173.

J. Grainger and W.D Stevenson. Power system analysis. McGraw-Hill, 1994.

G. J. Hegglid. An adaptive multivariable control system for hydroelectric generating units. Modeling, Identification and Control, 4(2):63, 1983.

IEEE. Standard definitions for excitation systems for synchronous machines. IEEE Std 421.1-2007, pages 1-33, July 2007.

P. Kundur. Power System Stability and Control. McGraw-Hill Professional, 1994. ISBN 007035958X.

M. Larsson. Coordinated Voltage Control in Electric Power Systems. phdthesis, Lund University, 2000.

J. M. Maciejowski. Predictive control with constraints. Pearson education, 2002.

R. Sharma. Predictive control with implementation, lecture notes. Telemark University College. Porsgrunn, Norway., 2014.

Statnett. FIKS Funksjonskrav i Kraftsystemet/Functional requirements in the power system. Technical report, Statnett, 2012.

T. Øyvang, D. Winkler, B. Lie, and G.J. Hegglid. Power system stability using modelica. In Proceedings of the 55th Conference on Simulation and Modelling (SIMS 55), Modelling, Simulation and Optimization, 21-22 October 2014, Aalborg, Denmark, number 108, pages 120-127. Linköping University Electronic Press, Linköpings universitet, 2014. 